\title{
A deeply annotated testbed for geographical text analysis
}

\author{
The Corpus of Lake District Writing
}

\author{
Paul Rayson \\ Lancaster University \\ Chris Donaldson \\ Lancaster University
}

\author{
Alex Reinhold \\ Lancaster University \\ Ian Gregory \\ Lancaster University
}

\author{
James Butler \\ Lancaster University \\ Joanna Taylor \\ Lancaster University
}

\begin{abstract}
This paper describes the development of an annotated corpus which forms a challenging testbed for geographical text analysis methods. This dataset, the Corpus of Lake District Writing (CLDW), consists of 80 manually digitised and annotated texts (comprising over 1.5 million word tokens). These texts were originally composed between 1622 and 1900, and they represent a range of different genres and authors. Collectively, the texts in the CLDW constitute an indicative sample of writing about the English Lake District during the early seventeenth century and the early twentieth century. The corpus is annotated more deeply than is currently possible with vanilla Named Entity Recognition, Disambiguation and geoparsing. This is especially true of the geographical information the corpus contains, since we have undertaken not only to link different historical and spelling variants of place-names, but also to identify and to differentiate geographical features such as waterfalls, woodlands, farms or inns. In addition, we illustrate the potential of the corpus as a gold standard by evaluating the results of three different NLP libraries and geoparsers on its contents. In the evaluation, the standard NER processing of the text by the different NLP libraries produces many false positive and false negative results, showing the strength of the gold standard.
\end{abstract}

\section{CCS CONCEPTS}

- Computing methodologies $\rightarrow$ Information extraction;

\section{KEYWORDS}

spatial humanities, corpus, toponyms, onomastics

\section{ACM Reference format:}

Paul Rayson, Alex Reinhold, James Butler, Chris Donaldson, Ian Gregory, and Joanna Taylor. 2017. A deeply annotated testbed for geographical text analysis. In Proceedings of 1st ACM SIGSPATIAL Workshop on Geospatial Humanities, Los Angeles Area, CA, USA, November 7-10, 2017 (GeoHumanities'17), 7 pages.

https://doi.org/10.1145/3149858.3149865

\begin{tabular}{l} 
(c) $\underset{\mathbf{E Y}}{\text { (i) }}$ \\
This work is licensed under a Creative Commons \\
\hline Attribution International 4.0 License.
\end{tabular}

GeoHumanities'17, November 7-10, 2017, Los Angeles Area, CA, USA

(C) 2017 Copyright held by the owner/author(s).

ACM ISBN 978-1-4503-5496-7/17/11.

https://doi.org/10.1145/3149858.3149865

\section{INTRODUCTION}

There has been an appreciable intensification of interest in geographical concepts and methods in the humanities over the past decade. This intensification, which has frequently been described as a 'spatial turn', has ushered in a renewed interest in mapping and in the use of the affordances of geographical information systems (GIS) to provide another dimension through which to explore and to interact with historical and modern data sources [14]. The developing field of Spatial Humanities has successfully exploited and enhanced such methods, and it has, moreover, modelled new approaches to the quantitative analysis of datasets to answer existing humanities research questions. By bringing such approaches together with computer science methods, such as Natural Language Processing (NLP), we have devised and implemented an innovative, semi-automated interpretive technique called geographical text analysis (GTA) to facilitate the examination of the qualitative elements of textual sources. GTA is a powerful, flexible and multifaceted process through which to explore humanities datasets. It enables scholars both to pose new research questions and to address old questions in ways that were not previously possible.

NLP methods for the automatic extraction of place-names from text data, known as Named Entity Recognition (NER) [9], have tended to focus on the identification of proper nouns through a variety of rule-based, statistical and machine-learning approaches. Such methods customarily classify the identified nouns according to high level categories: such as person, organisation, location or miscellaneous. The subsequent task of named entity disambiguation (NED) [4] links the identified terms to an existing knowledge base, such as Wikipedia or DBpedia, and thus to facilitate cross-referencing with other datasets and to enable connections with further metadata to be made. Similarly, toponym resolution is capable of supplying identified place-name entities with spatial information (such as coordinate data) that connects those place-name entities with a specific geographic location, using either spatial information [22] or textual context [27]. Joint models of named entity recognition and disambiguation have been proposed [17] recently, but these methods still target only high level categories.

The current state of the art in NER and NED has achieved promising results, and it has already facilitated significant research in the humanities [7, 8, 29]. Still, as this research has shown, there are challenges that need to be met in order for NLP methods to be fully and sustainably integrated in humanities scholarship. The challenges, as we see it, are at least three: Firstly, the emphasis that such methods place on the extraction of named entities via proper nouns alone can lead to the loss of much important information in corpora. In the case of NLP-based toponym resolution, this 
means that co-references to locations are often ignored. In order to overcome this limitation, we propose that instances of potentially ambiguous entities such as 'town', 'hill', or 'castle' could all be resolved to the nearest appropriate place-name occurring in the text, and their surrounding context taken into account when counting, mapping or collocating attributes linked to those places. Secondly, the coarse-grained nature of the location category customarily used in NLP is problematic and it often lacks detail sufficient to address many humanities research questions. Adding the ability to distinguish between different types of geographical features when extracting place-name entities can, we propose, enable separable analyses of, for instance, lakes, rivers, buildings or regions of widely differing geographical scopes, scales and spatial dimensions. Finally, especially in historical corpora, issues of simple spelling variation in place-names, to say nothing of archaic, obsolete and other sorts of place-name variations, can cause significant problems for conventional NER and NED methods, as these methods tend not to recognize non-standard spellings of place-name entities $[1,2,20]$. To rectify this problem, we propose that the modern standard spelling of a place-name might be used as a primary identifier (or 'parent'), and that all known alternative forms of that place-name be linked to this parent using a unique numeric identifier. In this paper, which emerges from research undertaken as part of the Geospatial Innovation In the Digital Humanities: A Deep Map of the Lake District project ${ }^{1}$, we elaborate on these problems and proposed solutions by providing a freely available and deeply manually annotated historical corpus resource called the Corpus of Lake District Writing (CLDW). This resource, as we explain below, is an ideal testbed for the development and application of future toponym resolution and geographical text analysis methods.

\section{BACKGROUND}

\subsection{The Lake District Deep Mapping project}

One of the aims of the Geospatial Innovation project, is to explore how we can use geographical information systems to explore and to investigate the geographies within textual corpora. GIS are a wellestablished technology that effectively act as a combination of a database management system, visualisation (mapping) and analysis platform for spatially referenced data $[13,16]$. GIS traditionally represent quantitative data; their data model involves having a table of attribute data that is similar to a traditional database table. Within the GIS, however, each row of attribute data is linked to an item of spatial data; namely, a graphical feature such as a point, which, when shown on a map, represents where the data are located. This combination of spatial and attribute data allows the researcher to examine and to pose questions about the geography within their dataset.

Our challenge was to explore how the basic data model of GIS could be used to analyse textual corpora. To do this, we needed to move from a collection of unstructured texts to a format that could be represented within the GIS. This required us to format our texts into a tabular structure in which each row contained a place-name, relevant attribute data and some form of spatial data to indicate the area on the map to which the place-name in question

\footnotetext{
${ }^{1}$ http://wp.lancs.ac.uk/lakesdeepmap/
}

referred. Place-names provide the link between geography in the text and locations on the map. The first stage in making the texts suitable for inclusion within GIS is thus to use NLP techniques to find the place-names. These place-names then need to be matched to coordinates taken from a gazetteer to provide locations. When performed automatically, this process is known as geo-parsing $[12,25]$. At the end of this process, the coordinates can be used as spatial data and the cotext (the text immediately surrounding the place-name) can be used as attribute data, along with other information from either the text, its metadata or other datasets, such as gazetteers.

In practice, one is unlikely to want to map all of the place-names within a corpus. More often, one will want to determine what places are associated with a particular idea or theme. To do this, only the place-names that occur within a set span of a search term need to be mapped. Thus, for example, we have used this approach to identify and to spatially analyse locations in the English Lake District that were historically associated with specific aesthetic ideals, such as beauty, sublimity and picturesqueness by mapping all of the place-names that occurred within \pm 10 words of the searchterms 'beautiful', 'sublime' and 'picturesque' $[8,10]$. Performing this analysis enabled us to test hypotheses about the historical meaning and application of these terms, and, in the process, to create a broader basis for the study of historical landscape aesthetics. We have also used a similar approach in field of historical demography in order to evaluate the places associated with particular diseases in public health reports [19,21].

There are, however, a number of potential sources of error in this process, and, following Unwin [28], we take error to include inaccuracy as well as genuine mistakes. The three major potential sources of this error lie with:

(1) Place-name identification errors: in some cases, words that are place-names may not be identified as such; in other cases, words that are not place-names may be wrongly identified as being place-names. This is problematic because NLP approaches can struggle to distinguish between, for example, Lancaster (a place), Stuart Lancaster (a person), a Lancaster (an aeroplane) or the Duke of Lancaster (a person or a pub) [23].

(2) Locational errors: a place-name may not be match to the correct location. This can be either because of disambiguation errors (Lancaster England, Lancaster Pennsylvania, or Lancaster California) or because of the difficulties that attend precisely representing features such as rivers, lakes or regions using point data.

(3) Span errors: a small span between the place-name and the search-term may lead to place-names that should be associated with the search-term being wrongly excluded. A larger span can reduce such errors, but it can also lead to other place-names being wrongly associated with the search-term.

\subsection{Related work}

A number of recent papers have highlighted the urgent need for open access corpora to be made available for toponym resolution, and a small number of corpora are becoming freely available containing manually verified locations. An early corpus, the TR-CoNLL 
[15] consists of 946 Reuters news stories totalling around 204,000 words, dating from 1996, with all toponyms manually referenced. As part of the Perseus Digital Library [3], the 19th Century American Collection totals some 58 million words, where toponyms were assigned automatically but corrected by hand. Relatedly, the War of the Rebellion corpus is annotated by hand with both multipoint and polygon geometries and contains two subsets: document geolocation (1.7 million words) and toponym resolution ( 0.48 million words) [6]. Other work has used Wikipedia corpora to resolve toponyms also at the article level [27], and the Wikipedia Toponym Retrieval (WikToR) corpus was created and geotagged programmatically and consists of 5000 unique Wiki pages [11]. All of these existing corpora suffer to a greater or lesser extent from the problems highlighted in section 1 where non-place-names are not taken into account, coarse-grained location categories are not able to answer humanities research questions and place-name variants are not resolved to one location.

\section{DATA}

Having the ability to consult such a variety of source material is important. One benefit of working with corpora composed of different kinds of historical materials is that these corpora can facilitate more contextually enriched investigations. Such corpora can enable researchers to evaluate and compare the information contained in a broad selection of historical sources simultaneously. In the process, they can both extend and enhance our understanding of specific themes, issues, attitudes and ideas.

The Corpus of Lake District Writing (CLDW) was assembled for the Lancaster University Spatial Humanities: Text, GIS \& Places project between 2012 and $2016^{2}$. The corpus contains 80 digitised texts about the Lake District. These texts date from between the years 1622 and 1900, and they include a number of different genres, such as travel journals, essays, novels and poetry. The authors of the texts in the CLDW are mostly male (six texts were written by women), and represent different nationalities including not only the UK, but also Germany and the United States. The 80 texts in the corpus contain over 1.5 million word tokens in total.

The corpus was run through the Edinburgh geoparser $[12]^{3}$, which performed Named Entity Recognition (NER) on the texts and matched locations to the Unlock gazetteer. Locations that were matched to the gazetteers were annotated with 'enamex' tags in the text that denoted the latitude and longitude of the location, as well as the source gazetteer, place-name and a confidence score. Locations found by NER that were not matched in the gazetteer were annotated with an 'enamex' tag without location information.

\subsection{Gold Standard Corpus}

In order to evaluate the original geoparser's performance, we conducted a case study using a gold standard subset of the corpus. This gold standard subset included a representative sample of 28 texts, which were selected from the different genres and historical periods included in the corpus (Table 1). Like the CLDW, this gold standard subset consists of texts from a range of different genres and authors that offer an indicative sample of writing about the

\footnotetext{
${ }^{2}$ http://www.lancaster.ac.uk/spatialhum/

${ }^{3}$ https://www.ltg.ed.ac.uk/software/geoparser
}

Lake District between the early seventeenth century and the early twentieth century.

The subset includes a diverse collection of travelogues and tourist guidebooks, as well as other kinds of topographical literature. Some of these texts are excerpts from larger works: for example Daniel Defoe's Tour thro' the Whole Island of Great Britain. The subset also includes entire texts that are more or less exclusively focused on the Lake District. Among these texts, there are works both by canonical authors, such as William Wordsworth and John Ruskin, and by authors whose writings are generally less well known, such as Priscilla Wakefield. Moreover, the gold standard subset includes works that were composed specifically for commercial publication, such as Wordsworth's Guide through the District of the Lakes, and printed versions of manuscript works that were not necessarily intended to appear in print, such as Lt. Hammond's Short Survey of 26 Counties and the letters of Samuel Taylor Coleridge and John Keats.

Quantitatively, the gold standard subset we compiled comprised approximately 242,000 word tokens: about one-sixth of the entire corpus. This subset was hand checked and coded using XML tags in order to mark every place-name entity it contained. These placename entities included the names of a variety of different regional, national and international locations, landmarks and geographical formations. As shown in the sample of text from page 550 of Daniel Defoe's Tour [5] inserted below, all of these identified place-name entities were marked-up with a customised tag (<cdplace $>)$ :

$<p>$ But I must not forget $<$ cdplace $>$ Winander Meer $<$ cdplace $>$, which makes the utmost northern bounds of this shire, which is famous for the char fish found here and hereabout, and no where else in $<$ cdplace $>$ England $</$ cdplace $>$; it is found indeed in some of the rivers or lakes in $<$ cdplace $>$ Swisserland $</$ cdplace $>$ among the $<c d-$ place $>$ Alps $</$ cdplace $>$, and some say in $<$ cdplace $>$ North Wales $</$ cdplace $>$; but I question the last. It is a curious fish, and, as a dainty, is potted, and sent far and near, as presents to the best friends. $</ p>$

As this example indicates, the texts in our gold standard subset contain references not only to a range of different national and international locations, but also to a variety of different spellings. Some of these spellings accord with the modern standardised spelling of specific place-names. In other cases, such as Winander Meer (an archaic name for Windermere), the spellings found in our corpus differ from the spellings conventionally used in modern gazetteers. The use of archaic spellings, such as Winander Meer, is both historically and onomastically significant in its own right [2], and so we were particularly keen to ensure that such variant placenames were correctly identified and tagged in our gold standard subset. Ensuring that such variants were recorded enabled us to compare how effective an off-the-shelf historical geoparser, such as the Edinburgh Geoparser, would be in working with the CLDW.

In previous research [24, 25] we employed an Early Modern English variant spelling detector (VARD) to match historical to modern forms, and the DEEP 'Historical Gazetteer of England's Place-Names' ${ }^{\prime 4}$ to improve coverage for the off-the-shelf geoparser. Based on this prior work we were able to determine that the Edinburgh Geoparser identified only 1277 of the 3718 place-name entities included in the Gold Standard subset. We were, moreover,

\footnotetext{
${ }^{4}$ http://www.placenames.org.uk/
} 
Table 1: Gold Standard Sample

\begin{tabular}{llrr}
\hline Author & Title & Date & Word tokens \\
\hline Lt. Hammond & A Relation of a Short Survey of 26 Counties & 1634 & 2,492 \\
Daniel Defoe & A Tour thro' the Whole Island of Great Britain & 1725 & 3,019 \\
George Smith & 'Survey of the Northwest Coast of England' & 1746 & 2,172 \\
George Smith & 'A Journey up to Cross-Fell Mountain' & 1747 & 2,774 \\
George Smith & 'A Journey to Caudbec Fells' & 1747 & 6,955 \\
John Brown & Description of the Lake and Vale at Keswick & 1751 & 1,107 \\
John Wesley & An Extract of the Rev. Mr. John Wesley's Journal & 1759 & 1,783 \\
Thomas Gray & Journal of A Visit to the Lake District in 1769 & 1769 & 6,388 \\
Thomas Pennant & A Tour in Scotland. MDCCLXIX & 1769 & 1,069 \\
Arthur Young & Six Months' Tour Through the North of England & 1770 & 13,388 \\
Thomas Pennant & A Tour in Scotland, and Voyage to the Hebrides & 1772 & 13,994 \\
Thomas West & A Guide to the Lakes & 1778 & 36,793 \\
William Cockin & Ode to the Genius of the Lakes. A Poem & 1780 & 4,793 \\
R. J. Sullivan & Observations Made During a Tour & 1780 & 2,799 \\
Stebbing Shaw & A Tour in 1787 & 1787 & 7,073 \\
J. H. Manners & Journal of a Tour & 1796 & 1,311 \\
S. T. Coleridge & Letters and Notebooks & 1802 & 9,237 \\
Priscilla Wakefield & A Family Tour through the British Empire & 1804 & 5,459 \\
Anonymous & Gleanings of a Wanderer & 1805 & 1,802 \\
John Keats & Letters & 1818 & 3,794 \\
William Wordsworth & The River Duddon: A Series of Sonnets & 1820 & 10,938 \\
Jonathan Otley & A Concise Description of the English Lakes & 1823 & 29,704 \\
John Ruskin & Iteriad; or, Three Weeks Among the Lakes & 1830 & 21,346 \\
William Wordsworth & Guide through the District of the Lakes & 1835 & 5,464 \\
Anonymous & Keswick and its Neighbourhood: A Hand-book & 1852 & 29,494 \\
James F. Clarke & Eleven Weeks in Europe & 1852 & 1,606 \\
Anonymous & The English Lakes & 1859 & 10,126 \\
Herbert Rix & 'Down the Duddon with Wordsworth' & 1893 & 5,412 \\
\hline & & Total & 242,292 \\
\hline & & & \\
\hline
\end{tabular}

able to establish that this variational rate was partially affected by the number and variety of variant spellings the subset contains. Some place-names, as we have recently reported elsewhere, were found to have up to 13 alternate names [2]. These findings indicate the potential margin of error on automated place-name recognition alone, before the issue of accurate geospatial identification can be considered. For instance, some illustrative examples of spelling variation found in the manual collection - but not by the original automated parsing - for Windermere include: Windermer Watter, Wynandermere, Windthemere Watther, Wiandermer, Winander Water, Win Dermere. None of these variants were identified by the automated geoparser, and the omission of these entries would have a significant deleterious effect on data that connected to those spelling forms to any degree.

\subsection{Deep analysis}

The comparatively small size of the full, 80-text corpus subsequently permitted the development of a manually curated collection of placename variants, including those affected by typographical issues (such as line breaks, erroneous transcriptions or printer errors). This was undertaken with corrected transcriptions of each text, with any formatting related to previously identified place-names removed to minimise any potential bias. This database took the modern spelling as the primary identifier (or 'parent'). Each was assigned a unique numeric identifier (specific to the corpus) and all alternate forms were linked to this parent. We assigned each entity identified as a valid identifiable and named location with two additional metadata components: first, whether it was internal or external to the Lake District; second, a geospatial category that indicated the type of place. The former was a binary value that used the modern national park boundaries, and the latter was a consideration designed to enhance the original online corpus resource, The Geotext Explorer ${ }^{5}$, to facilitate scholarly exploration of the corpus texts. Through the delineation of locational type, place-names with a common element (such as Coniston [lake], Coniston Old Man [height], Coniston Beck [waterway], Coniston Fell [height], Coniston [settlement] or Coniston Hall [house]) could be rendered as distinct entities, allowing an unprecedented range of geospatial analysis without any ambiguity as to point of reference. This process is similar to that found in the Pelagios Project, linking multiple variants and place classification within a text, although their focus is on the

\footnotetext{
${ }^{5}$ http://www.lancaster.ac.uk/fass/projects/spatialhum/geotext/
} 
classical world and historical gazetteers from a much earlier time period [26].

Nineteen geospatial categories were determined to provide an optimum range that offered sufficient distinction for geographical analysis without being too definitionally specific; for example, mountains, hills and ranges were all grouped together under the label 'Heights'. The categories chosen as most suited for the corpus were:

- Country. - Region. - Settlement. - Height. - Lake. - Waterway. Waterfall. - Vale. - Woodland. - Island. - Pass. - Specific. - Feature. House. - Farm. - Inn. - Street. - Battlesite. - Poetic.

Many of the labels are self-explanatory and were determined as being the most relevant to the environmental and journal-led discourse that comprised the corpus texts. Although the definitional range of several categories could be deemed too narrow for the specialised landscape analysis of other regions or time periods (for example, the presence of named valleys and estates would not be expected to feature as prominently in texts outside the scope of the CLDW), the use of narrower categorical identifiers makes combining an easier process for potential future development or integration into other resources. There is no standardised markup of format for expressing topographic form, and the categories listed above were deemed sufficiently distinct and important to the texts to merit inclusion for potential deep-analysis in authorial expression of these locational types. The 'Specific' category comprised named areas of specific tourist interest (e.g. viewing stations), including man-made elements (such as castle ruins, stone circles, churches or bridges), effectively covering locations which did not fall under any of the environmental archetypes. 'Features' were distinctly identified elements on larger named parent entities (e.g. Scafell Chimney, notable objects like the Bowder Stone, and named edges or ridges). Only sites assigned a proper name were included in the custom gazetteer.

The two metadata components allow for versatile deep analyses, permitting users to explore the data from a range of geospatial perspectives. These categories can, for example, be used to determine linguistic preferences, patterns, or relative strengths of referential relationships; that is, they might identify environmental aspects that are frequently compared or noted alongside one another in the corpus - whether in the same sentence, same paragraph, or any other custom range - and if these comparisons were to other sites in the region, or to other locations of renown. The assignment of geospatial labels mean such investigations are not restricted solely to individually named sites, but may be conducted on a broader topographic level that permits more detailed mapping of the routes and descriptions employed by different authors or genres. Such mapping endeavours require the highest level of accuracy in named entity geospatial identification.

\section{CASE STUDY: NER EVALUATION}

To test the potential of the corpus as a deeply annotated testbed we used two further well-known NLP libraries: Stanford CoreNLP [18] and OpenNLP ${ }^{6}$. These libraries were used to process the corpus with NER to detect place-names. Both libraries were used with the standard english models and default parameters. On top of this,

\footnotetext{
${ }^{6}$ https://opennlp.apache.org/
}

Table 2: Average NER Precision and Recall results for 28 files

\begin{tabular}{lrr}
\hline & Average Precision & Average Recall \\
\hline Edinburgh & 0.74 & 0.76 \\
Stanford & 0.90 & 0.57 \\
OpenNLP & 0.91 & 0.55 \\
\hline
\end{tabular}

we built a custom geoparsing engine that could take the results from the automatic processing; our geoparser allows one or more gazetteers to be added to the system. For this test of the corpus, we used the GeoNames gazetteer ${ }^{7}$ of the UK as a baseline. Our geoparser looks at each place-name and identifies every potential match in the gazetteer using three types of matches: exact match, inner match and outer match. In an exact match, the two terms being compared are identical, though not case sensitive. The inner match marries the place-names term with part of the gazetteer entry. (For instance, the place-name 'Britain' would match to 'Great Britain'.) An outer match is the reserve of the inner match (i.e. the place-name 'Great Britain' would match to 'Britain'). Each match type is given a confidence rating based on the Levenshtein distance calculation, which calculates the number of changes between two terms (e.g. exact matches return 0, and 'Rydal' compared to 'Rydal Mount' returns 6). In addition to the confidence rating, since the corpus is focused on the Lake District, our geoparser puts a priority on locations closer to that region.

After processing the corpus through each of the NLP libraries and our geoparser, we created a comparison tool to compare the gold standard text to the automatically processed text. The comparison tool looks at how many locations were identified in the gold standard and from each NLP library. It then looks at the amount of locations identified as the same location, and the amount identified as a different location. The results of this comparison across the whole corpus shows that the OpenNLP library identified the fewest place-names in the texts, and the gold standard identified the most place-names. The Stanford library and GeoNames Gazetteer matched $49 \%$ of the identified locations with the equivalent location in the gold standard. Figure 1 shows the comparison of the three automatic NER tools for all 80 files plus the manually corrected gold standard dataset for 28 files.

This baseline comparison of the gold standard corpus to three NLP libraries and Geonames gazetteer shows that the processing and manual correction of the corpus has produced a solid gold standard. While the processing of the corpus through the Edinburgh Geoparser had left a few false positives, the manual correction of the data has caught many of these, and has corrected inaccurate gazetteer matches. By adding historic gazetteers to our geoparser, the results of place-name geolocation should improve in future processing. Table 2 shows the average NER precision and recall scores across the 28 texts for the three tools, and figure 2 shows the detailed NER results per text in the gold standard corpus. As can be seen, both Stanford and OpenNLP prefer precision over recall, whereas the Edinburgh tool put more equal emphasis on them. All tools exhibit significant variation between the 28 texts in the gold standard.

\footnotetext{
${ }^{7}$ http://www.geonames.org/
} 


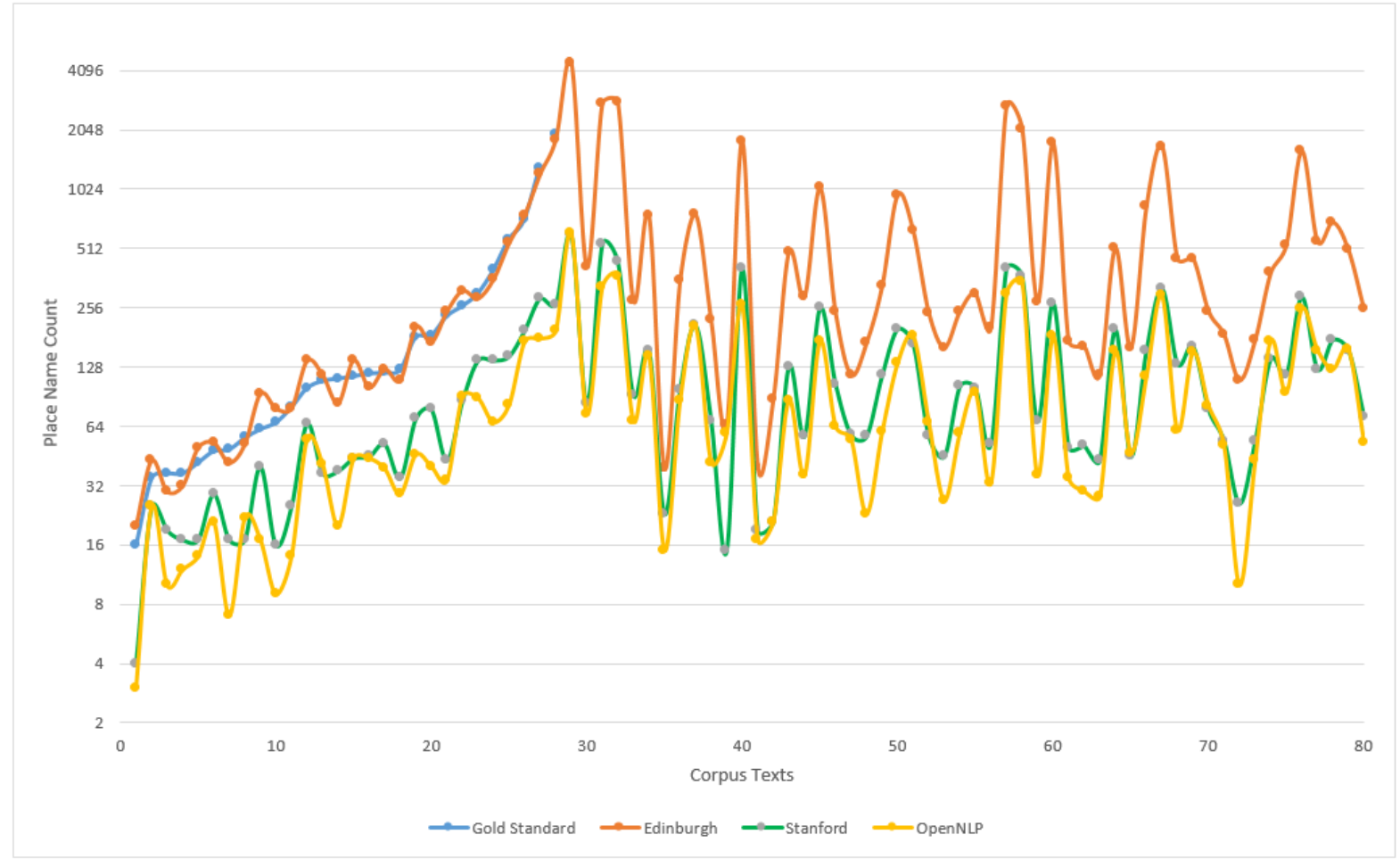

Figure 1: Comparison of NER tools with gold standard.

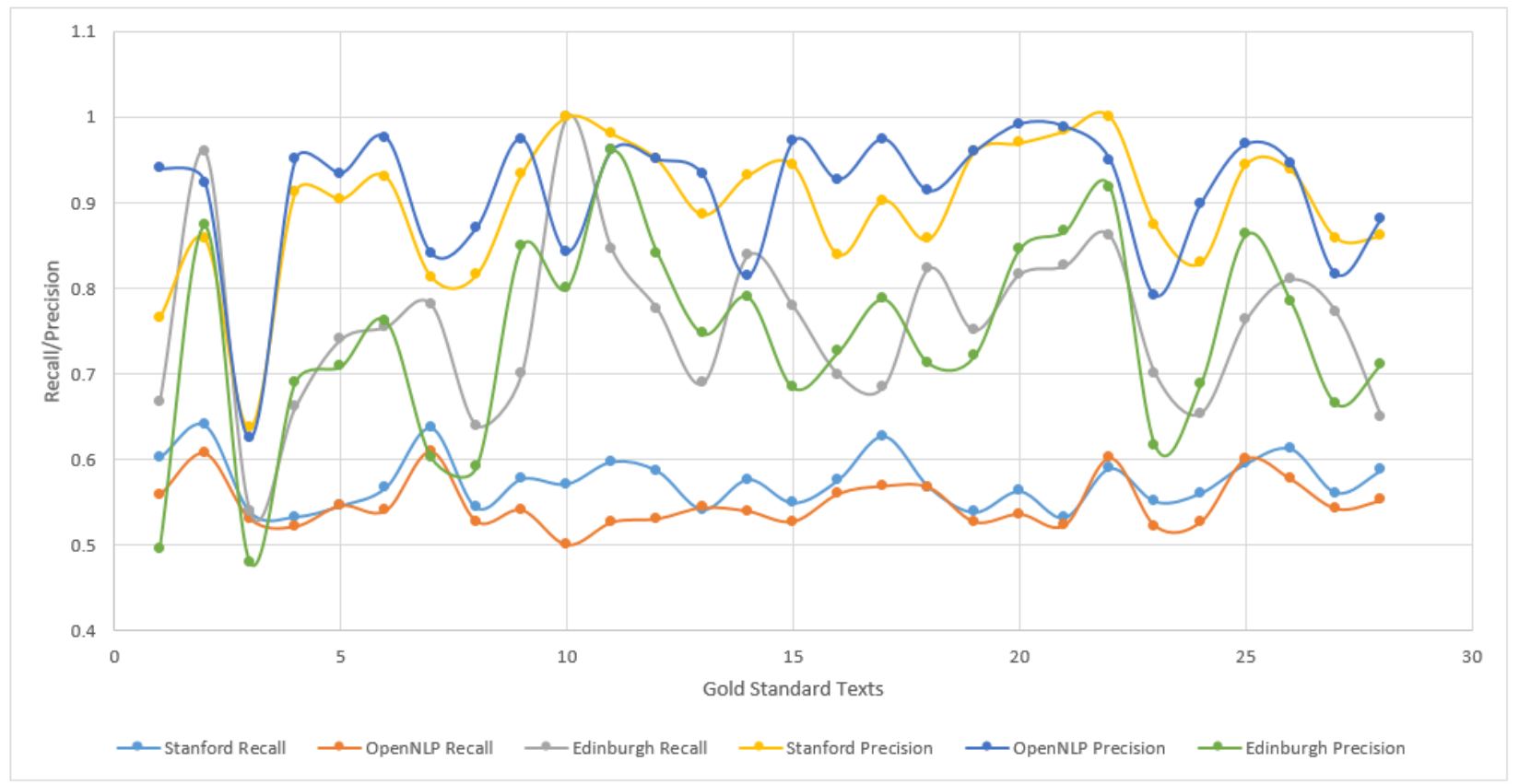

Figure 2: NER Precision and Recall results for 28 files. 


\section{CONCLUSION}

A number of recent NLP papers have highlighted the urgent need for more freely available toponym corpora in order to support development efforts in NER, NED and geoparsing research. In this paper, we have also provided additional requirements from humanities research questions, related to going beyond proper nouns as a basis for place-name references, the coarse-grained nature of the location category, and variation in terms of spelling and obsolete place-names referring to the same location over time. We have described the Corpus of Lake District Writing which goes some way to addressing these issues and provides a novel more deeply annotated testbed than has been available before. Much more research remains as future challenges for geographical text analysis, not least related to metonymy which occurs where a place-name is used as a substitute for something else. For example, in "Washington's war of words", 'Washington' is a metonym for the US Government. In addition, in the future, we plan to investigate online hosting for the gazetteer with URIs for ease of community reuse and linking. Our Lake District corpus is available to download from our GitHub repository https://github.com/UCREL/LakeDistrictCorpus.

\section{ACKNOWLEDGMENTS}

We would like to acknowledge the support of three projects, and their funders and members that have contributed to this research. Original explorations into mapping two textual accounts of journeys through the landscape of the Lake District began in the Mapping the Lakes: Towards a Literary GIS project funded by the British Academy, involving Dr David Cooper, now at Manchester Metropolitan University. The CLDW was created in the Spatial Humanities: Texts, GIS, places' research project which was funded by the European Research Council (ERC) under the European Union's Seventh Framework Programme (FP7/2007-2013) (agreement number 283850) from 2012-16. Finally, we are extending the corpus research in the Geospatial Innovation in the Digital Humanities: A Deep Map of the Lake District, which is funded by a Leverhulme Trust Research Project Grant (RPG-2015-230) from 2015-18. We also acknowledge the contribution of a number of research assistants who contributed to the preparation of the Corpus of Lake District Writing: Karen Donnelly, Joel Evans, Sayeed Ferdous, Chris Fletcher, Rachael Holland, Ann-Kathrin Marchlewski, Annegret Nissen, Amanda Pullan, Eliza Skakel, Enrico Torre, Tereza Valny, Alex Wilkinson, and Lynsey Wood.

\section{REFERENCES}

[1] Mariona Coll Ardanuy and Caroline Sporleder. 2017. Toponym Disambiguation in Historical Documents Using Semantic and Geographic Features. In Proceedings of the 2nd International Conference on Digital Access to Textual Cultural Heritage (DATeCH2017). ACM, New York, NY, USA, 175-180. https://doi.org/10.1145/ 3078081.3078099

[2] James O. Butler, Christopher E. Donaldson, Joanna E. Taylor, and Ian N. Gregory. 2017. Alts, Abbreviations, and AKAs: Historical Onomastic Variation and Automated Named Entity Recognition. Journal of Map \& Geography Libraries 13, 1 (2017), 58-81. https://doi.org/10.1080/15420353.2017.1307304

[3] Gregory Crane. 2000. The Perseus Digital Library. (2000). http://www.perseus tufts.edu/

[4] Silviu Cucerzan. 2007. Large-Scale Named Entity Disambiguation Based on Wikipedia Data. In Proceedings of EMNLP-CoNLL. http://aclanthology.coli. uni-saarland.de/pdf/D/D07/D07-1074.pdf

[5] Daniel Defoe. 1983. A Tour thro' the Whole Island of Great Britain (1724-26), ed. by Pat Rogers. Penguin.
[6] Grant DeLozier, Ben Wing, Jason Baldridge, and Scott Nesbit. 2016. Creating a Novel Geolocation Corpus from Historical Texts. In Proceedings of LAWX - The 10th Linguistic Annotation Workshop. Association for Computational Linguistics, 188-198. https://doi.org/10.18653/v1/W16-1721

[7] Caleb Derven, Aja Teehan, and John Keating. 2014. Mapping and unmapping Joyce: Geoparsing wandering rocks. In In Proceedings of Digital Humanities 2014.

[8] Christopher Donaldson, Ian N. Gregory, and Joanna E. Taylor. 2017. Locating the beautiful, picturesque, sublime and majestic: spatially analysing the application of aesthetic terminology in descriptions of the English Lake District. Fournal of Historical Geography 56 (2017), 43 - 60. https://doi.org/10.1016/j.jhg.2017.01.006

[9] Jenny Rose Finkel and Christopher D. Manning. 2009. Joint Parsing and Named Entity Recognition. In Proceedings of Human Language Technologies: The 2009 Annual Conference of the North American Chapter of the Association for Computational Linguistics. Association for Computational Linguistics, 326-334. http://aclanthology.coli.uni-saarland.de/pdf/N/N09/N09-1037.pdf

[10] Ian Gregory and Christopher Donaldson. 2016. Geographical text analysis: digital cartographies of Lake District literature. In Literary mapping in the digital age, David Cooper, Chris Donaldson, and Patricia Murrieta-Flores (Eds.). Routledge, 67-78.

[11] Milan Gritta, Mohammad Taher Pilehvar, Nut Limsopatham, and Nigel Collier. 2017. What's missing in geographical parsing? Language Resources and Evaluation (07 Mar 2017). https://doi.org/10.1007/s10579-017-9385-8

[12] Claire Grover, Richard Tobin, Kate Byrne, Matthew Woollard, James Reid, Stuart Dunn, and Julian Ball. 2010. Use of the Edinburgh geoparser for georeferencing digitized historical collections. Philosophical Transactions of the Royal Society of London A: Mathematical, Physical and Engineering Sciences 368, 1925 (2010), 3875-3889. https://doi.org/10.1098/rsta.2010.0149

[13] I. Heywood, S. Cornelius, and S. Carver. 2002. An Introduction to Geographical Information Systems. 2nd edition. Prentice Hall.

[14] R.T. Tally Jr. 2013. Spatiality. Routledge.

[15] Jochen L Leidner. 2008. Toponym Resolution in Text : Annotation, Evaluation and Applications of Spatial Grounding of Place Names. Universal Press, FL, USA.

[16] P.A. Longley, M.F. Goodchild, D.J. Maguire, and D.W. Rhind. 2001. Geographical Information Systems and Science. Wiley.

[17] Gang Luo, Xiaojiang Huang, Chin-Yew Lin, and Zaiqing Nie. 2015. Joint Entity Recognition and Disambiguation. In Proceedings of the 2015 Conference on Empirical Methods in Natural Language Processing. Association for Computational Linguistics, 879-888. https://doi.org/10.18653/v1/D15-1104

[18] Christopher D. Manning, Mihai Surdeanu, John Bauer, Jenny Finkel, Steven J. Bethard, and David McClosky. 2014. The Stanford CoreNLP Natural Language Processing Toolkit. In Association for Computational Linguistics (ACL) System Demonstrations. 55-60. http://www.aclweb.org/anthology/P/P14/P14-5010

[19] Patricia Murrieta-Flores, Alistair Baron, Ian Gregory, Andrew Hardie, and Paul Rayson. 2015. Automatically analysing large texts in a GIS environment: the Registrar General's reports and cholera in the nineteenth century. Transactions in GIS 19, 2 (4 2015), 296-320. https://doi.org/10.1111/tgis.12106

[20] Paolo Plini, Sabina Di Franco, and Rosamaria Salvatori. 2016. One name one place? Dealing with toponyms in WWI. Geofournal (21 Nov 2016). https://doi. org/10.1007/s10708-016-9760-9

[21] Catherine Porter, Paul Atkinson, and Ian Gregory. 2015. Geographical text analysis: a new approach to understanding nineteenth-century mortality. Health and Place 36 (11 2015), 25-34. https://doi.org/10.1016/j.healthplace.2015.08.010

[22] Erik Rauch, Michael Bukatin, and Kenneth Baker. 2003. A confidence-based framework for disambiguating geographic terms. (2003). http://aclanthology.coli. uni-saarland.de/pdf/W/W03/W03-0108.pdf

[23] Paul Rayson, Alistair Baron, and Andrew Hardie. 2012. Which 'Lancaster' do you mean? Disambiguation challenges in extracting place names for Spatial Humanities. In Proceedings of the Digital Humanities Congress, Sheffield University.

[24] C. J. Rupp, Paul Rayson, Alistair Baron, Christopher Donaldson, Ian Gregory, Andrew Hardie, and Patricia Murrieta-Flores. 2013. Customising geoparsing and georeferencing for historical texts. IEEE, 59-62. https://doi.org/10.1109/BigData. 2013.6691671

[25] C. J. Rupp, Paul Rayson, Ian Gregory, Andrew Hardie, Amelia Joulain, and Daniel Hartmann. 2014. Dealing with heterogeneous big data when geoparsing historical corpora. In Proceedings of the 2014 IEEE International Conference on Big Data. IEEE, 80-83. https://doi.org/10.1109/BigData.2014.7004457

[26] Barker E. Simon, R. and L. Isaksen. 2012. Exploring Pelagios: a visual browser for geo-tagged datasets. Agirre et al. (eds.), International Workshop on Supporting Users' Exploration of Digital Libraries. (2012), 29-34.

[27] Michael Speriosu and Jason Baldridge. 2013. Text-Driven Toponym Resolution using Indirect Supervision. In Proceedings of the 51st Annual Meeting of the Association for Computational Linguistics. ACL, 1466-1476. http://aclanthology. coli.uni-saarland.de/pdf/P/P13/P13-1144.pdf

[28] David J Unwin. 1995. Geographical information systems and the problem of'error and uncertainty'. Progress in Human Geography 19, 4 (1995), 549-558.

[29] Matthew Wilkens. 2013. The Geographic Imagination of Civil War-Era American Fiction. American Literary History 25, 4 (2013), 803-840. https://doi.org/10.1093/ alh/ajt045 